\title{
The application of dynamic scanning in target detection and imaging based on an NR-PC flat lens
}

\author{
MENG WenJuan ${ }^{1 *}$, CAO Hui ${ }^{2}$, SHEN TingGen ${ }^{1,2}$, WANG FeiFei ${ }^{2}$, LI ZhengHua $^{3}$, \\ YUAN BaoGuo ${ }^{2}$, HUANG Ming ${ }^{1} \&$ LIAN YingFei ${ }^{2}$ \\ ${ }^{1}$ Department of Telecommunication Engineering, Jiangsu University, Zhenjiang 212013, China; \\ ${ }^{2}$ Institute of Applied Physics, Jiangsu University, Zhenjiang 212013, China; \\ ${ }^{3}$ Communication T\&R Section, Zhenjiang Watercraft College, Zhenjiang 212013, China
}

Received August 26, 2010; accepted November 30, 2010

\begin{abstract}
In this paper a finite-difference time-domain method is used to model and analyze the application of dynamic scanning in target detection and imaging by using an effective negative-refraction photonic crystal (NR-PC) flat lens. The results show that there is a transmission peak, with a value far greater than unity, resulting from the influence of mini-forbidden bands and resonance excitation effect at a resonance frequency of $0.3068(a / \lambda)$. Thus, the lightwave emitted from the point source will provide strong backscattered waves after being focused on the target by the NR-PC lens that greatly improves the refocusing resolution and imaging resolution of the backscattered wave. Furthermore, a comparison with a non-dynamic scanning scheme clearly demonstrates that the dynamic scanning scheme provides improved refocusing resolution. In conclusion, our investigation optimized the performance of a detection and imaging system, and provided the basis for converting an idealized LHM lens into a physically realizable NR-PC flat lens.
\end{abstract}

negative effective index, dynamic scanning, scattering signal, refocusing resolution

Citation: Meng W J, Cao H, Shen T G, et al. The application of dynamic scanning in target detection and imaging based on an NR-PC flat lens. Chinese Sci Bull, 2011, 56: 830-834, doi: 10.1007/s11434-010-4334-6

Initiated by the recent microwave experiment demonstration [1] of Smith et al. on the unique electromagnetic properties of Veselago's negative-refraction-index materials (NRIM) [2], there has been a progressive increase in experiments to verify these unique properties [3,4]. Because the NRIM have negative permittivity and permeability, and the electric and magnetic field vectors form a left-handed set with the wave vector, such artificial materials are also referred to as left handed materials (LHM).

The realization of the LHM has provoked great interest in many specific applications, such as in the area of near-field target detection and imaging. Among these applications of LHM, focusing with lenses that have flat surfaces has been proposed [5]. Theoretical analysis and nu-

*Corresponding author (email: mengujs2010@gmail.com) merical simulations [6] indicated that the so-called perfect lens made of LHM with no losses may achieve a focus resolution overcoming the optical diffraction limit. Generally, higher focus resolution yields better imaging resolution [7].

Though, in theory, it is quite reasonable to use an LHM flat lens for high-resolution near-field target detection and imaging [7], there is still much uncertainty about whether such materials actually exist in nature. Using different methods, Notomi has demonstrated 2D Photonic crystals exhibiting negative index or negative refraction effects [8], namely negative refraction photonic crystals (NR-PC). From these results we know that negative refraction usually occurs in the band gap of the equifrequency surface (EFS) in $k$ space because the size of the contour of the EFS in the $k$ space decreases with increasing frequencies. The contour of 
the EFS up to a certain frequency takes a quasi-circular shape [9], which means the light propagating in the PC is similar to that propagating in the isotropic medium at these frequencies. Hence, the effective negative index of refraction $\left(n_{\mathrm{eff}}\right)$ can be used to describe the propagation property of light with frequencies falling into a certain frequency spectrum for a given NR-PC, which could be used as a flat lens in near field target detection and imaging [10,11].

The two-dimensional photonic crystal structure examined in this paper, as shown in Figure 1(a), is formed by periodically drilling 7 rows (along the $Z$-axis) of 30 identical air holes (along the $X$-axis) in a GaAs matrix with a dielectric constant $\varepsilon=12.96(n=3.6)$. The air cylinders take on a triangular array, and the radius of the air cylinders is $0.4 a$ ( $a$ represents the lattice constant). The refractive index of the photonic crystal is calculated for the TM mode and is drawn in Figure 1(b) using the algorithm in reference [8]. It is clear that $n_{\text {eff }}$ changes with normalized frequency $\omega=a / \lambda$. From Figure 1(b), we know that when $n_{\text {eff }}$ takes the value of -1 , the corresponding normalized frequency $\omega$ is about 0.3068 .

Based on the phenomenon of negative refraction, it is easy to envisage that the NR-PC flat lens and the LHM flat lens [5] share the same image-forming principles. As shown in Figure 1(a), the lightwave emitted from a point source on one side of the NR-PC lens can be focused at one focal point $F_{1}$ inside the NR-PC lens, and then at the other focal point $F_{2}$ outside the NR-PC lens. When a target is brought into the focal point $F_{2}$, the lightwave from the source is focused on the target by the flat NR-PC lens, and the target will backscatter the focused lightwave, which will then be refocused in the vicinity of the source point by the same flat NR-PC lens. Because the focal point $F_{2}$ can be controlled easily by moving the point source along the lines of the surface of the lens or adjusting the distance between them, the target can easily be scanned and its image in the vicinity of the source point is significantly enhanced. Generally, the complete lightwave recorded at each receiving point is the combination of three parts, i.e. the wave emitted from the source, the wave reflected from the entrance and exit sur- faces of the NR-PC lens (for NR-PC of $n_{\text {eff }} \neq-1$ ), and the refocused wave of the backscattered lightwave (scattering signal). Thus, the scattering signal is acquired by taking the difference between what is recorded by the detector with or without a target on the focal point $F_{2}$. This means that target detection and imaging can be realized, with a high resolution ratio and without a complicated imaging algorithm, simply by computing the lightwave distribution of the scattering signal from the target [6].

In this paper, we mainly discuss the target detection and imaging properties of the dynamic scanning system by using the NR-PC flat lens. Numerical simulations with the 2D finite-difference time-domain (FDTD) method show that a sharp transmission peak of the lightwave appears at the resonance frequency $0.3068(a / \lambda)$ for the NR-PC flat lens. In addition, the lightwave backscattered from the target is enhanced greatly, which significantly improves the lateral refocusing and imaging resolution, and as a result optimizes the performance of the target detection and imaging system. The design and calculation models of the NR-PC flat lens are further studied using the dynamic scanning scheme. Detailed comparison with non-dynamic scanning will be more helpful in evaluating a specific application of the dynamic scanning scheme on target detection and imaging.

\section{Design and calculation models of the NR-PC flat lens}

The Maxwell's equations in the photonic crystals can be written as

$$
\begin{gathered}
\frac{\partial \boldsymbol{H}}{\partial t}=-\frac{1}{\mu_{0} \mu(r)} \nabla \times \boldsymbol{E}, \\
\frac{\partial \boldsymbol{E}}{\partial t}=\frac{1}{\varepsilon_{0} \varepsilon(r)} \nabla \times \boldsymbol{H},
\end{gathered}
$$

where $\nabla=\frac{\partial}{\partial x} \boldsymbol{i}+\frac{\partial}{\partial y} \boldsymbol{j}+\frac{\partial}{\partial z} \boldsymbol{k}$ is the Hamiltonian operator, $\boldsymbol{E}$
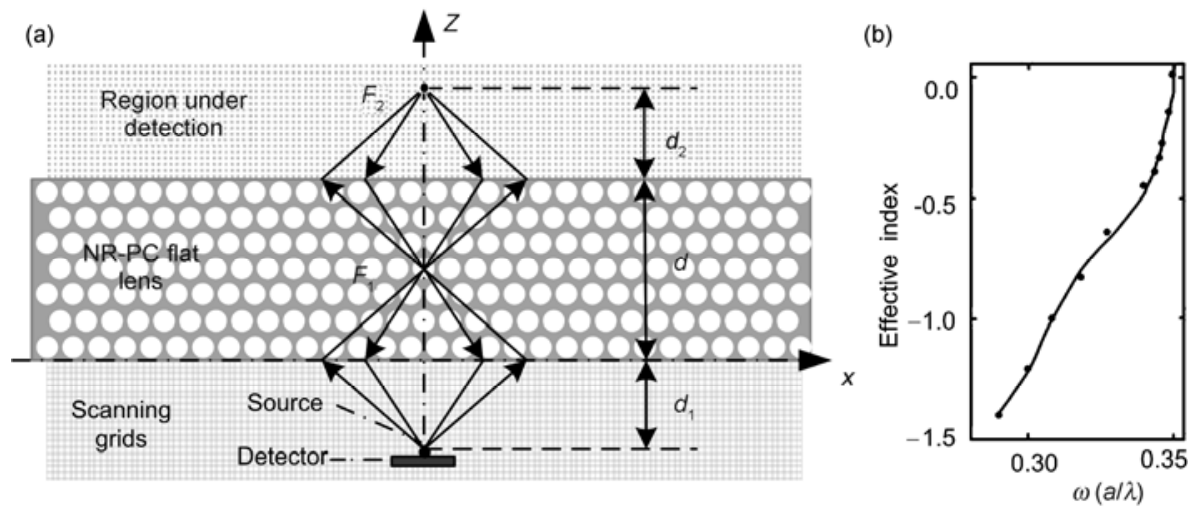

Figure 1 (a) Target detection and imaging using the NR-PC flat lens; (b) change curve of $n_{\text {eff }}$ and $\omega$ in the NR-PC lens (for the TM mode) [8]. 
and $\boldsymbol{H}$ are the electric and magnetic field vectors of the electromagnetic wave, the permittivity $\varepsilon(r)$ is the relative dielectric constant, and $\mu(r)=1$ is the relative magnetic permeability.

The FDTD method [12] is well-established with a somewhat lower computational capacity in comparison to its high accuracy when compared with other methods. It has been widely used to study characteristics of electromagnetic waves in NR-PC. Its fundamental principle is that Maxwell's equations are first expressed as scalar equations of electric and magnetic field components in Cartesian coordinates, and then the differential quotient is replaced with the difference quotient with accuracy to the second order. In our simulation, a perfectly matched layer (PML) is used in the $X$ and $Y$ directions as boundary conditions [12]. Because these equations are functions of space and time, they can be discretized in the space and time domains by the Yee-cell technique and be used to find field solutions numerically.

It is noteworthy that the mode of light propagating in photonic crystals (PC) is very different from that in LHM. For the LHM with refractive index $n=-1$, there is no reflection at the air-LHM interface, however light will experience multiple reflection and refraction at the air-PC interface, even for NR-PC when $n_{\text {eff }}=-1$, leading to great losses or much lower transmissivity for the light propagating through the NR-PC. To optimize the performance of the focus-scanning scheme, an effective way to improve transmissivity is needed.

The corresponding investigation of raising the transmissivity has already been presented in our previous thesis cited by OPTIK [13]. When the center frequency $\left(\omega_{\mathrm{p}}\right)$ of the wave source is set at $0.3068(a / \lambda)$, the transmission is enhanced dramatically, with the transmission coefficient up to 4500 at the frequency point of $0.3068(a / \lambda)$. The physical mechanism can be explained by the redistribution of optical energy. The incident optical waves with a different frequency will experience intensive Bragg scattering when they are incident upon the NR-PC and propagating in the NR-PC because of the periodic distribution of negative-refraction media, which results in mini-forbidden bands and a photonic tunneling effect for a given NR-PC $[14,15]$. At the same time, the optical energy is highly localized, and the high transmissivity appears at the resonance frequency.

\section{Refocusing of the backscattered wave in target detection and imaging from an NR-PC flat lens}

The focusing-refocusing property is a very important performance parameter that supports the use of the NR-PC flat lens for lightwave target detection and imaging. By taking the measured width at 0.707 -maximum of the normalized field intensity of the refocused beam profile as the definition of resolution [16], the performance of the target detection and imaging system based on the NR-PC flat lens can be further evaluated. In particular, for the detection and imaging of a small target at an early stage, high sensitivity is very desirable, thus, our investigation may have great significance for imaging systems.

In our FDTD simulations, the center frequency of the point source is adjusted to $0.3068(a / \lambda)$ for a high transmissivity. Meanwhile, we consider the typical situation where a point source and a detector are set to move together along the scanning line $z=-\lambda$ with intervals of $\Delta x=0.2 \mu \mathrm{m}$. The $2 \mathrm{D}$ flat NR-PC lens with a thickness of $d=2 \lambda$ is set at $0 \leqslant z$ $\leqslant 2 \lambda$, and a target of a PEC square with a side length of $L=$ $1 / 3 \lambda$ is located at the focal point $F_{2}$.

First the performance of the complete lightwave is investigated. The simulation diagram of the complete lightwave field in the computation area and its corresponding field intensity distribution along the scanning line $z=-\lambda$ are depicted in Figure 2(a) and (b).

From the arrowheaded lines in Figure 2(a), it is clear that the imaging of the NR-PC lens obeys geometrical optics. Furthermore, there is a one-to-one correspondence between the energy distribution of the complete lightwave field (Figure 2(a)) and the distribution curve of its field intensity (Figure 2(b)), and the maximum field intensity on the line $z$ $=-\lambda$ is obtained in the vicinity of the point source.

Figure 2 depicts the properties of the complete lightwave, whereas the main role of the NR-PC flat lens in target detection and imaging is actually embodied in the characteristics of the scattering signal from the target. Therefore, to obtain the scattering wave, we may substrate the fields recorded without the target at $F_{2}$ from the fields recorded with the target at $F_{2}$. Then, on the basis that the other parameters

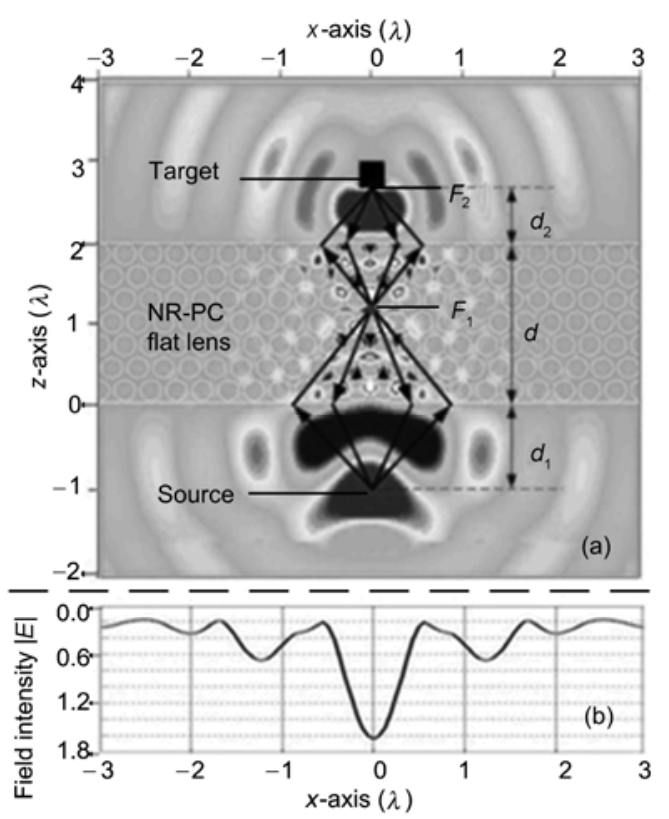

Figure 2 (a) Simulation diagram of the complete lightwave in the computation area; (b) beam profile of the complete lightwave field intensity along line $z=-\lambda$. 
are the same as previously, we further contrast the properties of the scattering signal with those obtained when $\omega_{\mathrm{p}}=$ $0.2068(a / \lambda)$. Detailed comparisons are shown in Figure 3.

By measuring the full width of the beam profile at 0.707-maximum as given in Figure 3, we find that the refocusing resolutions are approximately $0.3718 \lambda$ and $1.59 \lambda$ for $\omega_{\mathrm{p}}=0.3068(a / \lambda)$ and $\omega_{\mathrm{p}}=0.2068(a / \lambda)$. Obviously, the lateral refocusing resolution corresponding to $0.3068(a / \lambda)$ is four times that of $0.2068(a / \lambda)$. This is clear from the theoretical point of view in which the scattering signal of the target is greatly enhanced because the backscattered wave has a higher transmissivity in the narrow band around the frequency of $0.3068(a / \lambda)$. This results in significant enhancement of the lateral refocusing and imaging resolution, and optimizes the performance of the focus-scanning scheme. We now see that the concept of a flat NR-PC lens is physically sound and experimentally feasible.

\section{Importance of dynamic scanning in target detection and imaging using NR-PC flat lens}

It should be noted that our research outlined above was based on the dynamic scanning scheme. This is different from the non-dynamic scanning scheme with the point source being set to stay static and only the detector moving along the scanning line. The dynamic scanning scheme requires the point source and the detector to move together. Further comparisons between the two scanning schemes will be more helpful for evaluating the superiority of the dynamic scanning scheme in target detection and imaging.

In the following simulation, we consider the above defined flat NR-PC lens $d=2 \lambda$ thick and the point source with $\omega_{\mathrm{p}}=0.3068(a / \lambda)$. Moreover, the square PEC target at focal point $F_{2}$ with side lengths of $L=1 / 6 \lambda, L=1 / 10 \lambda$ and $L=$ $1 / 30 \lambda$ are detected separately using the two scanning schemes. The corresponding beam profiles of the normalized field intensity of the scattering signals are presented in Figure 4. As shown in Figure 4, the solid curves indicate the

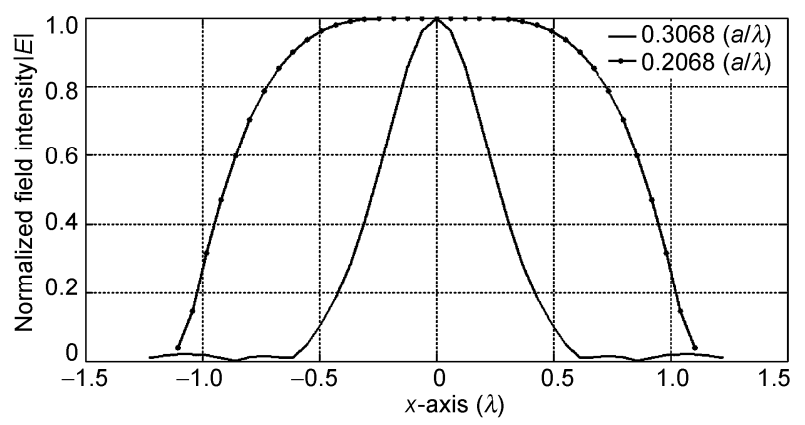

Figure 3 Beam profile of the normalized field intensity of the scattering signal from the square PEC target with a side length of $L=1 / 3 \lambda$. The point source with a center frequency of $0.2068(a / \lambda)$ and $0.3068(a / \lambda)$ is localized at $(-\lambda, 0)$, respectively.

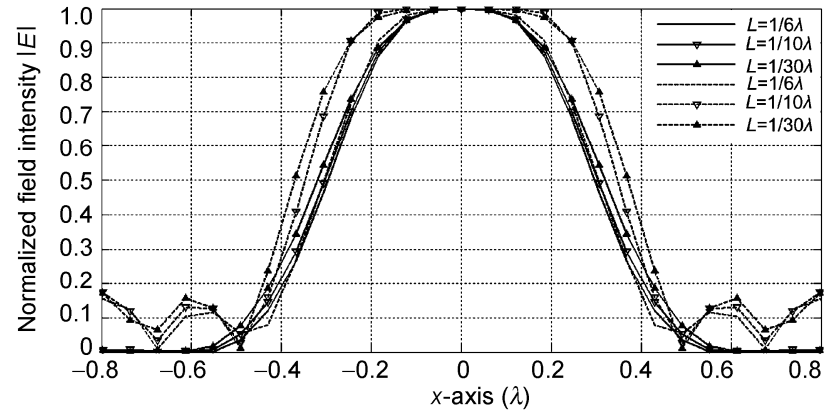

Figure 4 Beam profiles of the normalized field intensity of the scattering signal obtained by detecting different square PEC targets with the dynamic (the solid curves) and non-dynamic (the dashed curves) scanning schemes.

Table 1 Refocusing resolutions obtained by detecting different square PEC targets with the dynamic and non-dynamic scanning schemes

\begin{tabular}{lllc}
\hline & \multicolumn{3}{c}{ The size of the square targets } \\
\cline { 2 - 4 } & $L=1 / 6 \lambda$ & $L=1 / 10 \lambda$ & $L=1 / 30 \lambda$ \\
\hline $\begin{array}{l}\text { Refocusing resolution } \\
\text { for dynamic scanning }\end{array}$ & $0.4736 \lambda$ & $0.4868 \lambda$ & $0.51 \lambda$ \\
\hline $\begin{array}{l}\text { Refocusing resolution } \\
\text { for non-dynamic scanning }\end{array}$ & $0.5004 \lambda$ & $0.6014 \lambda$ & $0.6388 \lambda$ \\
\hline
\end{tabular}

dynamic scanning scheme, and the dashed curves depict the non-dynamic scanning scheme.

From Figure 4 and Table 1 we know that the dynamic scanning scheme may achieve better refocusing resolution than the non-dynamic scanning scheme in target detection and imaging. When using the dynamic scanning scheme to detect a square target with side lengths of $L=1 / 6 \lambda, L=$ $1 / 10 \lambda$ and $L=1 / 30 \lambda$, there were approximately $6 \%, 24 \%$ and $25 \%$ improvements in the refocusing resolution, when compared to those when the non-dynamic scanning scheme was used. In other words, decreasing the size of the target results in significant improvements to the superiority of the dynamic scanning scheme. In addition, regardless of which scanning scheme is chosen, the refocusing resolution improves with the increase in the target size, which obviously conforms to the general laws of physics.

To further demonstrate the important role of dynamic scanning in target detection and imaging, we changed the shape of the targets by applying cylinder PEC targets with diameters of $D=1 / 6 \lambda, D=1 / 10 \lambda$ and $D=1 / 30 \lambda$. Relevant refocusing properties after comparing with those of the square targets are presented in Figure 5 and Table 2. As shown in Figure 5, the solid curves indicate the situation where the square targets were under detection, while the dashed curves depict the situation when the cylinder targets were used.

According to [7], for the LHM lens, the dynamic scanning scheme has a scanning resolution of $0.257 \lambda$ when detecting a PEC cylinder target with a diameter of $D=1 / 6 \lambda$. For the NR-PC lens, the refocusing resolution was approximately $0.2564 \lambda$, which is nearly equal to that of the LHM lens. Therefore, the NR-PC flat lens and the LHM 


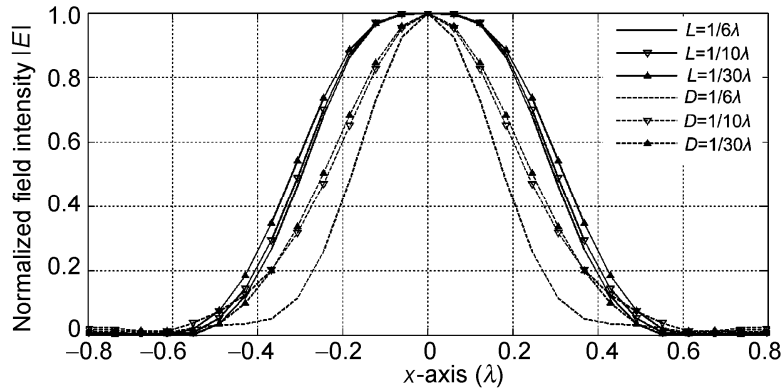

Figure 5 Beam profiles of the normalized field intensity of the scattering signal obtained by detecting different square (the solid curves) and cylinder (the dashed curves) PEC targets with the dynamic scanning scheme.

Table 2 Refocusing resolutions obtained by detecting different square and cylinder PEC targets with the dynamic scanning scheme

\begin{tabular}{ccc}
\hline & Size of targets & Resolution \\
\hline \multirow{3}{*}{ Square target } & $L=1 / 6 \lambda$ & $0.4736 \lambda$ \\
& $L=1 / 10 \lambda$ & $0.4868 \lambda$ \\
& $L=1 / 30 \lambda$ & $0.51 \lambda$ \\
\hline \multirow{3}{*}{ Cylinder target } & $D=1 / 6 \lambda$ & $0.2564 \lambda$ \\
& $D=1 / 10 \lambda$ & $0.3286 \lambda$ \\
& $D=1 / 30 \lambda$ & $0.3518 \lambda$ \\
\hline
\end{tabular}

lens have approximately equal image accuracy when using the dynamic scanning scheme. From Figure 5 and Table 2 we know that the refocusing resolution obtained by the detection of the cylinder target is far superior to that of the square target.

\section{Conclusions}

On the basis of the 2D FDTD method, we applied a dynamic scanning scheme to study the characteristics of the NR-PC flat lens. It was demonstrated that because of the influence of the mini-forbidden band and resonance excitation effect, high transmissivity will appear at the resonance frequency of $0.3068(a / \lambda)$ when the lightwave goes through the NR-PC lens. In addition, the focusing characteristics of the NR-PC lens and the exponential amplification of the evanescent wave [5] make the scheme quite efficient in raising the backscattered wave, which leads to a refocusing resolution and imaging resolution with significant enhancement. The detailed performance analysis demonstrated that the dynamic scanning scheme is superior to the nondynamic scanning scheme in target detection and imaging. Furthermore, the NR-PC flat lens and the LHM lens have approximately equal image accuracy when using the dynamic scanning scheme, and the refocusing resolution obtained by the detection of a cylinder target is greatly superior to that of a square target.

This work was supported by 2010 Jiangsu University Hundred Undergraduate Innovation Project (2010073).

1 Veselago V G. The electrodynamics of substances with simultaneously negative values of $\varepsilon$ and $\mu$. Sov Phys Usp, 1968, 10: 509-514

2 Shelby R A, Smith D R, Schultz S. Experimental verification of a negative index of refraction. Science, 2001, 292: 77-79

3 Zhao Q, Zhao X P, Kang L, et al. Reflection and phase of left-handed metamaterials at microwave frequencies. Chinese Sci Bull, 2005, 50: 395-398

4 Cao Y J, Wen G J, Wu K M, et al. A novel approach to design microwave medium of negative refractive index and simulation verification. Chinese Sci Bull, 2007, 52: 433-439

5 Pendry J B. Negative refraction makes a perfect lens. Phys Rev Lett, 2000, 85: 3966-3969

6 Rao X S, Ong C K. Sub-wavelength imaging by a left-handed material superlens. Phys Rev E, 2003, 68: 067601-067603

7 Wang G, Fang J R, Dong X T. Resolution of near-field microwave target detection and imaging by using flat LHM lens. IEEE Trans Antennas Propog, 2007, 55: 3534-3541

8 Notomi M. Theory of light propagation in strongly modulated photonic crystals: Refraction like behavior in the vicinity of the photonic band gap. Phys Rev B, 2000, 62: 10696-10706

9 Guven K, Aydin K, Alici K B, et al. Spectral negative refraction and focusing analysis of a two-dimensional left-handed photonic crystal lens. Phys Rev B, 2004, 70: 205125-205130

10 Fang Y T, Sun H J, Shen T G. New evidences of negative refraction in photonic crystals. Opt Mater, 2006, 28: 1156-1159

11 Fang Y T, Shen T G. Multi-imaging by photonic crystal slab using negative refraction. Chin Phy Lett, 2005, 22: 949-951

12 Yee K S. Numerical solution of initial boundary value problems involving Maxwell's equations in isotropic media. IEEE Trans Antennas Propog, 1966, 14: 302-308

13 Li Z H, Xue Y L, Deng Z Q, et al. Study on optical switching effect of photonic crystals with negative effective index of refraction. Optik, 2009, 120: 605-609

14 Sharon A, Rosenblatt D, Friesem A A. Narrow spectral bandwidths with grating waveguide structures. Appl Phys Lett, 1996, 69: 4154-4156

15 Shadrivov I V, Sukhorukov A A, Kivshar Y S, et al. Nonlinear surface waves in left-handed materials. Phys Rev E, 2004, 69: 16617-16619

16 Grbic A, Eleftheriades G V. Overcoming the diffraction limit with a planar left-handed transmission-line lens. Phys Rev Lett, 2004, 92: 117403-117406

Open Access This article is distributed under the terms of the Creative Commons Attribution License which permits any use, distribution, and reproduction in any medium, provided the original author(s) and source are credited. 